observed. With a great many observations the anticyclonic inflow can be brought out by a system of averaging, as shown in the American Meteorological Journal for August I893. H. HeLM CLAYTON.

Blue Hill Meteorological Observatory, June I7.

\section{Effects of a Lightning Flash in Ben Nevis Observatory.}

Whenever a thunder-storm passes the summit of the Ben, there occurs almost invariably a discharge from metallic bodies in the Observatory, as the cloud is passing away. A flash of greater or less extent is given off the stoves, accompanied by a sharp crack. In January I 890 there was an exceptionally severe flash; "one of the observers was almost knocked down when sitting writing, and the telegraph wire was fused, and all communication stopped for five days." But more destructive than any previous flash was that which occurred this year on June I9, when the Observatory narrowly escaped being destroyed by fire. Between two and three o'clock on that afternoon, repeated clicks on the telegraph instrument were heard by one of the assistants who was sitting in the office; he had been carefully noting the times at which the clicks occurred, when suddenly the whole office was filled with a brilliant flash and deafening roar. A pillar of smoke was discharged from the telegraph instrument and from the stove-pipe, filling the room. So severe was the flash that the assistant, who was quite deafened by the report, thought that his hair had been singed. A second slighter discharge took place immediately after, when the writer had entered the office to commence the fifteen hours observations. The discharge hurled two boxes and a small picture, that were in the vicinity of the lightning protector, across the kitchen, and blew off the button and outer casement of the electric bell in the visitors' room. The solder on the kitchen chimney outside, a copper fastening of the lightning conductor, and many portions of the telegraphic wire and apparatus were fused, and the wood work of the Observatory was scorched in several places. The great flash occurred at I4 hours $57^{\frac{1}{2}}$ minutes, and the hourly barometric reading was taken at I 5 hours, as usual. There wa a very heavy fall of snow at the time, equivalent to 0.470 inches of rainfall for the hour, but in the confusion the writer omitted to take the rain-gauge with him, and had to return for it. This was a fortunate incident ; for it was only on leaving the office for a second time, that he observed smoke and flame issuing from behind the panelling between the kitchen and the office. Assistance was secured, and the fire-which was in a very awkward and dangerous place--was overcome in good time, and the damage done was very slight.

The damage done to the telegraphic apparatus, however, was serious, and Mr. Crompton, engineer of the Post Office telegraphs, has supplied me with the following information.

The lightning protector was badly fused, the plates showing a patch of fusion as large as a sixpence. This saved the cable from serious damage. All connecting wires within the building were rendered useless. The majority were so heated as to melt the insulation off, and, in one or two cases, the copper conductors were melted by the discharge. In one case, the fusion set fire to the woodwork.

The coils of Neale's sounder were fused and rendered useless. The keys suffered worst of all, the left pedal or "tapper" bearing the strongest evidence of the severity of the discharge. The back contact (platinum), the brass extension holding the same, and the steel spring (platinum-tipped) above, all being fused into one solid amalgam. The pillar, to which the zinc leading wire from the battery was connected, had a large patch of fusion near its base, and the front platinum contacts of the same (left-hand) pedal were consumed entirely. There were small traces of fusion on the right pedal, but of a trifling character. The line wire connected to the left-hand terminal of the coil had been fused close to the terminal. The interior of the instrument case was considerably blackened, as also the greater portion of the keys, as a result of the "arc" caused by the discharge at the moment of fusion.

The vacuum protector at Aslimtee, the base of the cable, also the plate protector in Fort William Post Office, were fused, but only slightly, the main discharge having expended itself on the summit. The Low Level Observatory instrument and protector were unimpaired, and communication between there and Fort William Post Office was carried on as usual after the removal of the fault in the Post Office protector.

The registering aneroid shows a slight upward kick at the NO. I 34 I, VOL. 52$]$ time, but the curve is otherwise fairly steady; the temperature was $3 I^{\circ} 7^{\circ} \mathrm{F}$, the wind south-south-east and light. Heavy snow was falling at the time, which, with a fall on the $\mathrm{I} 7 \mathrm{th}$, made a total depth of nine inches on the summit. St. Elmo's Fire was very strongly felt and heard until after seventeen hours.

Wili.iam S. BRUCE.

\section{The Kinetic Theory of Gases.}

IT seems to me that Mr. Burbury's and Prof. Boltzmann': last letters will enable us to reconcile all the main differences of opinion which were brought to light in our recent correspondence in the columns of NATURE. From Prof. Boltzmann's letter it appears that the Minimum Theorem can only be applied with absolute certainty to gases whose molecules are not too closely crowded together. Thus the proof that an aggregation of molecules tends continuously towards the Boltzmann-Maxwell distribution depends quite as much on assumptions as to the mixing of the molecules between collisions as on consideration of what happens at collisions. We cannot prove for certain that densely crowded assemblages of molecules such as solids and liquids tend to assume this distribution, and this is just as it should be, for when a substance is capable of existing simultaneously in two states, the distribution cannot be unique. For the same reason the proof does not apply to molecules moving about in a continuous medium such as the ether. So far from this limitation being a weak point in the proof, it precludes the theorem from proving too much, or from leading to results which may not accord with experience.

If we do not know that solids and liquids satisfy the Boltzmann-Maxwell distribution, we, nevertheless, know that they are subject to the Second Law of Thermodynamics. It cannot be said that any dynamical "proof of the Second Law" that has yet been given, is so conclusive as the mere statement of the Law itself, but the proof of the Minimum Theorem subject to "Condition (A)" leads to a result somewhat analogous to the statement that when two or more bodies at unequal temperature are brought into thermal contact, their entropy tends to increase. For let the probability of the coordinates on momenta of the molecules of one body lying between certain limits be proportional to $\mathrm{F}$ (all the coordinates and momenta being included in the multiple differential by which $\mathrm{F}$ is multiplied). Let the corresponding probability for a second body be proportional to $f$. Then when the two bodies are placed in thermal contact, we know of no relation connecting the two simultaneous probabilities, and we may therefore assume them to be independent, so that condition (A) is satisfied, at any rate initially. The theorem then asserts that at all subsequent instants of time, the value of the Minimum Function will be not greater than its initial value, and therefore it either remains stationary or decreases every time the process is repeated. Thus far we can get if no further.

The application of the Second Law depends largely on the distinction between available and unavailable energy. When we construct a thermodynamic engine for converting heat into work, we introduce just the kind of external disturbances that Mr. Burbury requires every time that the "working substance" is placed in contact with either the "source" or the refrigerator." G. H. BRYAN.

\section{An Abnormal Rose.}

I HAVE in my garden at Reigate a white Moss rose-tree, every blossom on which is white except one which is half white and half red, divided diametrically in nearly equal portions.

The colours are not shaded one into the other, but are perfectly distinct, and one petal is half red and half white, the edge of the colouration being quite sharp.

I am told that one similar blossom was produced earlier in the season.

I imagine this is an attempt to revert to its ancestral colour, but by what mechanism such a partial result has been accomplished seems difficult to understand.

\section{NeWnhaM Browne.}

THERE are several varieties of rose that sport or revert in the manner described by Mr. Newnham Browne. The "York and Lancaster" rose is a familiar example. In this, the recognised or genuine condition is red and white striped; but the proportions of white and red are rarely exactly the same in any two flowers. 